\title{
Optimal Control of Wind Turbines under Islanded Operation
}

\author{
Magdi S. Mahmoud, Mojeed O. Oyedeji \\ Systems Engineering Department, King Fahd University of Petroleum and Minerals, Dhahran, KSA \\ Email: msmahmoud@kfupm.edu.sa, msmahmoud,g201513510@kfupm.edu.sa
}

How to cite this paper: Mahmoud, M.S. and Oyedeji, M.O. (2017) Optimal Control of Wind Turbines under Islanded Operation. Intelligent Control and Automation, 8, 1-14.

https://doi.org/10.4236/ica.2017.81001

Received: October 5, 2016

Accepted: December 6, 2016

Published: December 9, 2016

Copyright $\odot 2017$ by authors and Scientific Research Publishing Inc. This work is licensed under the Creative Commons Attribution International License (CC BY 4.0).

http://creativecommons.org/licenses/by/4.0/

\begin{abstract}
In this paper, an optimal control scheme for wind turbine output torque and power regulation under the influence of wind disturbances is presented. The system considered is a dynamic mechanical-based model with pitch and generator torque actuators for controlling the pitch and generator torque. The performance of linear matrix inequality (LMI) formalism of linear quadratic regulator (LQR); linear quadratic regulator with integral action (LQRI) and model predictive control (MPC) were compared in response to a step change in wind disturbance. It is shown by Matlab simulation that the LQRI outperformed both LQR and MPC controllers.
\end{abstract}

\section{Keywords}

Wind Turbine, Linear Quadratic Regulator, Linear Quadratic Regulator with Integral Action, Model Predictive Control, Output Torque, Power Regulation

\section{Introduction}

Wind energy is one of the primitive sources of energy. The kinetic energy from the wind can be converted to mechanical energy using wind energy conversion systems. Wind energy conversion systems include: wind mill, wind pump and the modern-day wind turbine. The first practical windmill, called Sistan mill was developed in the 7th century in Iran. According to [1], windmills were used in the USA in the 1930's for electricity production and pumping of water and the first utility grid connected wind turbine was built by John Brown and Co. in 1951. Because more attention is being shifted to clean energy sources due to increasing environmental concerns, nations are contributing more to clean energy research and increasing the amount of power generated from the wind. The total capacity of wind power in the world at the end of 2014 was estimated around some $370 \mathrm{GW}$ and is estimated to reach $670 \mathrm{GW}$ by the end of 2019 [1]. 
Depending on the axis of rotation, wind turbines can be differentiated into two categories: Horizontal axis wind turbine (HAWT) and vertical axis wind turbine (VAWT) (see Figure 1). Compared with the VAWT, the HAWT has a higher wind energy conversion efficiency due to its blade design but it requires stronger tower support due to heavy weight of the nacelle and the cost of installation is higher compared with the VAWT. The operation of the VAWT is independent of the wind direction but has lower wind energy conversion efficiency and it is more susceptible to higher torque fluctuations and mechanical vibrations. It is commonly found in domestic/private installations where the energy demand is not so high.

Wind turbine systems can be classified into three basic system configurations typically used on wind farms depending on the type of generators used [1] [2] [3]: fixed-speed wind turbine (FSWT), variable-speed wind turbine (VSWT) [4] and variable-speed wind turbine with direct driven generators (VSWT-DDG). In the case of the FSWT, the squirrel-cage induction generator (SCIG) is connected directly to the grid using a multistage gearing system to match the rotor speed with the turbine. The doubly-fed induction generator (DFIG) is used in the VSWT, its stator windings are connected directly to the grid, while its rotor windings are connected to the grid using an electronic converter that utilizes thirty percents of the total generator capacity. In the VWST-DDG, a synchronous generator and a full-scale power electronic converter are used. Compared with other configurations, it does not need a multi-stage gearbox system because a low-speed high torque synchronous generator is used. The FSWT configurations have the advantage of simplicity, low cost and low maintenance but they have low energy conversion efficiency because they cannot take advantage of the increasing wind speed to improve their efficiency. The VSWT configurations have higher energy conversion efficiency but are characterized by large installa-

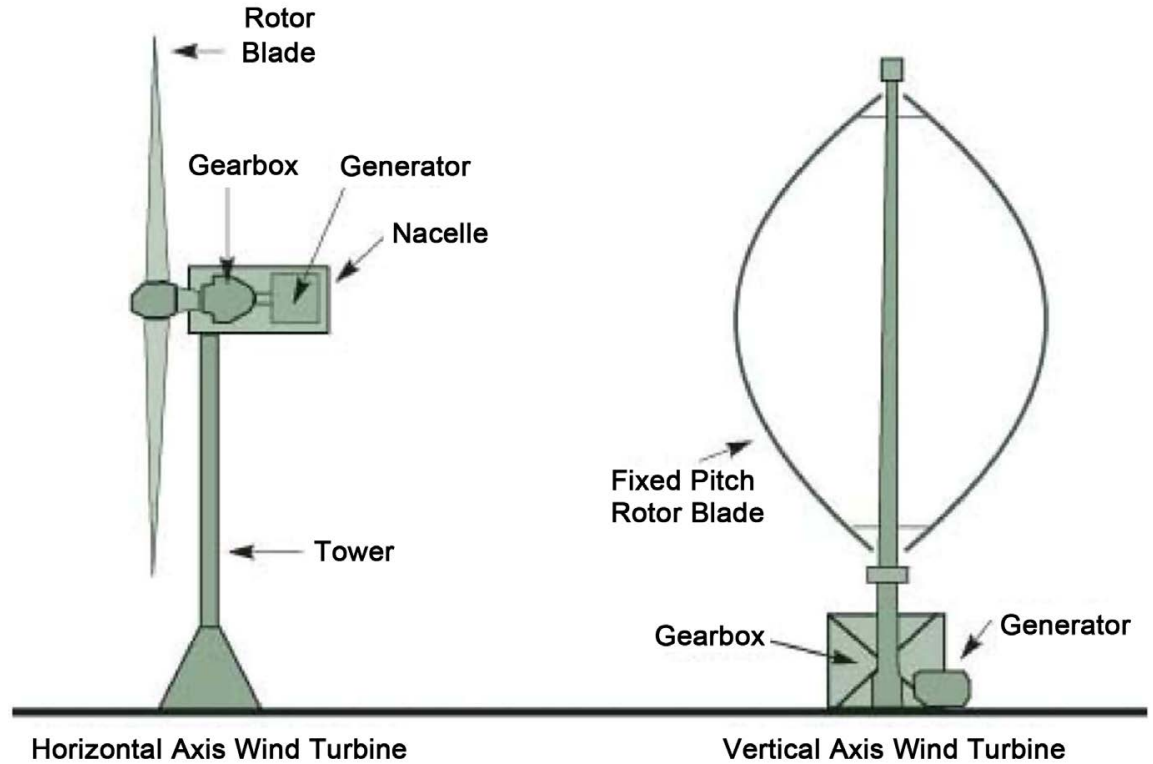

Figure 1. Components of a DFIG-based WTS. 
tion cost and highly complex control system. Dynamic modeling issues and control methods of wind turbine systems are presented in [5], [6] and [7] and the references cited therein.

In this paper, we contribute to the further development of control for a class of wind turbine systems by designing improved output torque and power regulation is face of wind disturbances. Several distinct control design approaches are considered:

- Linear quadratic control (LQR) design, for which we establish an LMI formalism yielding a static state-feedback gain.

- Linear quadratic control with Integral action (LQRI), for which we enhance the LQR design by adding an integral term.

- Model predictive control (MPC), for which we establish an LMI formalism yielding a static state-feedback gain.

- $H_{2}$ control design, for which we establish an LMI formalism based on the $H_{2}$-norm condition and yielding a dynamic-feedback structure and

- $H_{\infty}$ control design, for which we establish an LMI formalism based on the $H_{\infty}$-norm condition and yielding a dynamic-feedback structure.

All the design procedures are casted into the format of feasibility problem over linear matrix inequalities (LMIs). By this way, effective computational methods are established yielding guaranteed quality solution. Simulation studies are performed for all the approaches yielding good performance results.

\section{Modeling of Wind Turbine Systems}

Several wind turbine models exist depending on the objective of the modeling. Some models focus mainly on fault detection and identification while other models are control-oriented. Regardless of the objective, the main subsystems usually considered are: aerodynamics, drive train, generator and the grid (for grid connected systems).

\subsection{Aerodynamics Modeling}

The aerodynamics of the system is defined by nonlinear algebraic equations. The aerodynamic power of the turbine is expressed as [5]:

$$
\begin{gathered}
P=\frac{1}{2} \rho \pi R_{b}^{2} V_{w}^{3} C_{p}(\lambda, \beta) \\
C_{p}=0.73\left(\frac{151}{\lambda_{i}}-0.53 \beta-0.002 \beta^{2.14}-13.2\right) \mathrm{e}^{-\frac{18.4}{\lambda_{i}}} \\
\lambda_{i}=\left(\frac{1}{\lambda-0.02 \beta}-\frac{0.003}{\beta^{3}+1}\right)^{-1}
\end{gathered}
$$

where $\rho$ represents the air density, $R_{b}$ is the blade radius, $V_{w}$ is the wind velocity and the power coefficient $C_{p}$ is the power coefficient is a highly nonlinear function of $\lambda$. 


\subsection{Drive-Train Modeling}

The dynamics of the drive train can be represented with six-mass, three-mass, two-mass and one mass models. The six-mass model is considered most detailed and represents accurately the non-linear behavior of the drive-train. In the six-mass model, six different inertias are defined: the three different blade inertias, hub, gearbox, generator inertia. The three-mass model is can be obtained from the six-mass model by lumping the weights of the three blades and the hub together. In the two-mass model (2), one mass is used to represent the low speed turbine and the high speed generator. The connecting shaft is then modeled as a spring and damper. A simplified single-mass drive train model (3) can be obtained by removing the shaft stiffness and mutual damping from the two-mass model. A single-inertia is thus used to represent the whole system.

$$
\begin{gathered}
\dot{\omega}_{r}=\frac{Q_{r}}{K_{r}}-\frac{K_{s}}{J_{r}} \phi_{\Delta}-\frac{D_{s}}{J_{r}} \omega_{r}+\frac{D_{s}}{J_{r} N_{g}} \omega_{g} \\
\dot{\omega}_{g}=\frac{Q_{g}}{J_{g}}-\frac{K_{s}}{J_{g} N_{g}} \phi_{\Delta}-\frac{D_{s}}{J_{r} N_{g}} \omega_{r}+\frac{D_{s}}{J_{r} N_{g}^{2}} \omega_{g} \\
\dot{\phi}_{\Delta}=\omega_{r}-\frac{\omega_{g}}{N_{g}} \\
\dot{\omega}_{m}=-\frac{f}{J} \omega_{m}-T_{e m}+T_{m}
\end{gathered}
$$

\subsection{Generator Modeling}

The most common electrical generators connected studied in literature are the Permanent Magnet Synchronous Generator (PMSG) and the Doubly-Fed Induction Generator (DFIG). The nonlinear model of a DFIG-based wind turbine [8] in the dq-reference frame is given in (4), (5), (6) and (7).

$$
\begin{aligned}
& \dot{\phi}_{r d}=-\frac{R_{r}}{\sigma L_{r}} \phi_{r d}+\frac{R_{r} L_{m}}{\sigma L_{r} L_{s}} \phi_{s d}+\omega_{r} \phi_{r q}+V_{r d} \\
& \dot{\phi}_{r q}=-\frac{R_{r}}{\sigma L_{r}} \phi_{r q}+\frac{R_{r} L_{m}}{\sigma L_{r} L_{s}} \phi_{s q}-\omega_{r} \phi_{r d}+V_{r q} \\
& \dot{\phi}_{s d}=-\frac{R_{s}}{\sigma L_{s}} \phi_{s d}+\frac{R_{s} L_{m}}{\sigma L_{r} L_{s}} \phi_{r d}+\omega_{s} \phi_{s d}+V_{s d} \\
& \dot{\phi}_{s q}=-\frac{R_{s}}{\sigma L_{s}} \phi_{s q}+\frac{R_{s} L_{m}}{\sigma L_{r} L_{s}} \phi_{r q}-\omega_{s} \phi_{s d}+V_{s q}
\end{aligned}
$$

The dynamic nonlinear model of a PMSG-based wind turbine is described by [9]:

$$
\begin{gathered}
i_{s d}=-\frac{R_{s}}{L_{s d}} i_{s d}+\frac{L_{s q}}{L_{s d}} \omega_{d q} i_{s q}+\frac{V_{s d}}{L_{s d}} \\
\dot{i}_{s q}=-\frac{R_{s}}{L_{s d}} i_{s q}-\frac{L_{s q}}{L_{s d}} \omega_{d q} i_{s d}+\omega_{d q} \phi_{r d}+\frac{V_{s q}}{L_{s d}}
\end{gathered}
$$


Various combinations of the above dynamic models of each subsystem are possible. Models described in literature may be characterized as mechanicalbased or electrical-based models. The mechanical based models are more detailed in the model of the aerodynamics and drive train. These models are used for designing pitch and torque actuator controllers [10]. The electrical- based models are more detailed in the modeling of the electrical generators and the grid and usually use the two or one-mass representation of the drive train.

\section{Integrated System Model}

The linearized dynamic model of the system is defined by (10)-(11). The operating parameters of the system were obtained at $18 \mathrm{~m} / \mathrm{s}$ of wind velocity. The model consist of a two-mass dive drain, a pitch and generator torque actuator.

$$
\begin{aligned}
& \delta \dot{x}=A \delta x+B_{1} \delta u_{i n}+B_{2} \delta v_{i n} \\
& y=C \delta x \\
& A=\left[\begin{array}{cc}
A_{1} & 0 \\
0 & A_{2}
\end{array}\right], \quad B_{1}=\left[\begin{array}{cc}
B_{11} & 0 \\
0 & B_{22}
\end{array}\right], \\
& A_{1}=\left[\begin{array}{ccc}
\left.\frac{1}{J_{r}} \frac{\partial Q_{r}}{\partial \omega_{r}}\right|_{\bar{\omega}_{r}} & \frac{D_{s}}{J_{r} N_{g}} & \frac{-k_{s}}{J_{r}} \\
\frac{D_{s}}{J_{g} N_{g}} & \frac{D_{s}}{J_{g} N_{g}^{2}} & \frac{-k_{s}}{J_{g} N_{g}} \\
1 & \frac{1}{N_{g}} & 0
\end{array}\right], A_{2}=\left[\begin{array}{ccc}
-\frac{1}{\tau_{g}} & 0 & 0 \\
0 & 0 & 1 \\
0 & -\omega_{n}^{2} & -2 \zeta \omega_{n}
\end{array}\right], \\
& B_{12}=\left[\begin{array}{cc}
0 & 0 \\
\frac{1}{\tau_{g}} & 0 \\
0 & \omega_{n}^{2}
\end{array}\right], B_{11}=\left[\begin{array}{cc}
0 & \left.\frac{1}{J_{r}} \frac{\partial Q_{r}}{\partial \theta}\right|_{\bar{\theta}} \\
\frac{1}{J_{g}} & 0 \\
0 & 0
\end{array}\right] \\
& B_{2}=\left[\begin{array}{c}
B_{21} \\
0
\end{array}\right], B_{21}=\left[\begin{array}{cc}
\frac{1}{J_{r}} & \frac{\partial Q_{r}}{\partial v} \\
0 \\
0
\end{array} \mid\right. \\
& C=\left[\begin{array}{cccccc}
0 & \bar{Q}_{g} & 0 & \bar{\Omega}_{g} & 0 & 0 \\
1 & 0 & 0 & 0 & 0 & 0
\end{array}\right]
\end{aligned}
$$

where $x=\left[\omega_{r}, \omega_{g}, \phi, Q_{g}, \beta_{1}, \beta_{2}\right], u=\left[Q_{g}, \beta, Q_{\text {gref }}, \beta_{r e f}\right], \omega_{r}$ is the rotor/mechanical angular speed; $\omega_{g}$ is the generator angular speed; $\phi$ is the torsional speed; $Q_{g}$ is the generator torque; $\beta_{1}$ is the pitch displacement; $\beta_{2}$ is the pitch velocity. Letting $B=\left[B_{1} B_{2}\right], u=\left[\delta u_{i n} \delta v_{i n}\right]$, we cast the wind-turbine system into the compact form: 


$$
\begin{gathered}
\dot{x}=A x(t)+B u(t) \\
y=C x(t),
\end{gathered}
$$

Typical numerical values of the model parameters are presented in Table 1.

\section{Control Design of Wind Turbine Systems}

Now we look at the optimal control design and express all the design methods as feasibility problems over linear matrix inequalities (LMIs).

\subsection{LQR: Proportional Gain}

With focus on the linear-quadratic regulator (LQR) design, the associated quadratic cost function [11] is

$$
J=\int_{0}^{\infty}\left[y^{t}(t) Q y(t)+u^{t}(t) R u(t)\right] \mathrm{d} t
$$

where $0<Q, 0<R$ are output error and control weighting matrices. Initially, we present an LMI-based formulation to the LQ control of system (12) while minimizing the quadratic cost (13). We proceed to determine a linear optimal control $u=L x$ that achieves this goal.

Assumption 1. There exists a Lyapunov functional $V(x)$ which has the properties:

- $V(x)=x^{t} K x, K>0$

- There exists $\gamma_{+}>0$ such that $x_{0}^{t} K x_{0} \leq \gamma_{+}$

- $\dot{V}(x) \leq-\left[x^{t} C^{t} Q C x+u^{t} R u\right]$

Considering system (12) with linear control $u=L x$. The following theorem

\begin{tabular}{|c|c|}
\hline Parameter & Value \\
\hline$P_{e, \text { orm }}$ & $5000 \mathrm{KW}$ \\
\hline$\Omega_{r}$ & $12.1 \mathrm{rpm}$ \\
\hline$k_{s}$ & $867.637 \times 10^{6} \mathrm{~N} \cdot \mathrm{m} / \mathrm{rad}$ \\
\hline$D_{s}$ & $6.215 \times 10^{6} \mathrm{~N} \cdot \mathrm{m} /(\mathrm{rad} / \mathrm{s})$ \\
\hline$J_{g}$ & $534.116 \mathrm{~kg} / \mathrm{m}^{2}$ \\
\hline$J_{r}$ & $3.8768 \times 10^{7} \mathrm{~kg} / \mathrm{m}^{2}$ \\
\hline$J_{t}$ & $4.3792 \times 10^{7} \mathrm{~kg} / \mathrm{m}^{2}$ \\
\hline$R$ & $63 \mathrm{~m}$ \\
\hline$\eta$ & 1 \\
\hline$\tau_{g}$ & $0.1 \mathrm{~s}$ \\
\hline$\omega_{n}$ & $0.88 \mathrm{rad} / \mathrm{s}$ \\
\hline$\zeta$ & 0.9 \\
\hline
\end{tabular}
provides an LMI-based LQR design:

Table 1. NREL wind turbine data. 
Theorem 1 System (12) with the LQR control $u=L x$ is asymptotically stable and $J_{\infty} \leq V\left(x_{o}\right)$. Given matrices $Q>0, R>0$. If there exist matrices $S, Y$ such that

$\min _{\gamma_{+}, Y, S} \gamma_{+}$subject to

$$
\begin{aligned}
& {\left[\begin{array}{ccc}
(A Y+B S)+(A Y+B S)^{t} & Y C^{t} Q & Y L^{t} R \\
\bullet & -Q & 0 \\
\bullet & \bullet & -R
\end{array}\right]<0} \\
& {\left[\begin{array}{cc}
\gamma_{+} & x_{o}^{t} \\
\bullet & Y
\end{array}\right] \geq 0}
\end{aligned}
$$

has a feasible solution, then $L Q R$ gain matrix is $L=S Y^{-1}$.

Proof. By Assumption (1), and using control $u=L x$ in system (12), the inequality of the derivative of the Lyapunov functional is expressed as

$$
x^{t}\left[K(A+B L)+(A+B L)^{t} K^{t}\right] x \leq-x^{t}\left[C^{t} Q C+L^{t} R L\right] x
$$

It is evident that (16) is satisfied if there exists $L$ and $K$ such that

$$
\left[K(A+B L)+(A+B L)^{t} K^{t}\right]-\left[C^{t} Q C+L^{t} R L\right] \leq 0
$$

Simple computations on (13) in view of Assumption (1) yields $J_{\infty} \leq V\left(x_{0}\right)$. By minimizing the upper bound $\gamma_{+}$on the cost $x_{0}^{t} K x_{0}$, we obtain

$$
\min _{\gamma_{+}, K, L} \gamma_{+} \text {subject to (17) }
$$

To convexify the above problem, we first express (17) as

$$
\begin{gathered}
\Phi=K(A+B L)+(A+B L)^{t} K^{t} \\
\Pi=\left[\begin{array}{ccc}
\Phi & C^{t} Q & L^{t} R \\
\bullet & -Q & 0 \\
\bullet & \bullet & -R
\end{array}\right] \leq 0
\end{gathered}
$$

Pre- and post-multiply (19) by $\operatorname{diag}\{Y, I, I\}$ and using $Y=K^{-1}, S=L K^{-1}$ it yields (14). Additionally, the inequality bound of the Lyapunov functional can be expressed as

$$
\left[\begin{array}{cc}
\gamma_{+} & x_{0}^{t} \\
\bullet & K^{-1}
\end{array}\right] \geq 0
$$

which can be manipulated to yield (15). When a feasible solution is attained, we get $L=S Y^{-1}, K=Y^{-1}$ as desired.

\subsection{LQR: Proportional-Integral Gain}

A modified formulation of the LQR (13) is considered with an additional term in the cost representing the integral of the deviation of the output from its initial state $z(t)=\int_{0}^{t} y(\tau) \mathrm{d} \tau$. This formulation will be referred to as the LQRI.

$$
\tilde{J}=\int_{0}^{\infty}\left(y(\tau)^{t} y(\tau)+\rho u(\tau)^{t} u(\tau)+\sigma z(\tau)^{t} z(\tau)\right) \mathrm{d} \tau
$$


Treating $\dot{z}=C x$ as additional state variable, we define $\eta=\left[\begin{array}{ll}x & z\end{array}\right]^{t}$. The augmented system becomes

$$
\begin{gathered}
\dot{\eta}=\left[\begin{array}{ll}
A & 0 \\
C & 0
\end{array}\right] \eta+\left[\begin{array}{c}
B \\
0
\end{array}\right] u=\tilde{A} \eta+\tilde{B} u \\
\bar{y}=\left[\begin{array}{ll}
C & 0
\end{array}\right] \eta=\tilde{C} \eta
\end{gathered}
$$

and hence we can re-write (21) as:

$$
\begin{gathered}
\tilde{J}=\int_{0}^{\infty}\left[\eta^{t}(\tau) \tilde{Q} \eta(\tau)+u(\tau)^{t} \tilde{R} u(\tau)\right] \mathrm{d} \tau \\
\tilde{Q}=\left[\begin{array}{cc}
C^{t} C & 0 \\
0 & \sigma I
\end{array}\right], \tilde{R}=\rho I, \rho, \sigma>0
\end{gathered}
$$

Next, we present an LMI-based formulation to the LQI control of system (22) while minimizing the quadratic cost (23). We proceed to determine a linear optimal control $u=\tilde{L} x$ that achieves this goal.

Assumption 2. There exists a Lyapunov functional $\tilde{V}(x)$ which has the properties:

- $\tilde{V}(\eta)=\eta^{t} \bar{K} \eta, \bar{K}>0$,

- There exists $\gamma_{+}>0$ such that $\eta_{o}^{t} \bar{K} \eta_{o} \leq \gamma_{+}$

- $\dot{\tilde{V}}(\eta) \leq-\left[\eta^{t} \tilde{Q} \eta+u^{t} \tilde{R} u\right]$

The following theorem provides an LMI-based LQRI design for system (22) with linear control $u=\tilde{L} x$ :

Theorem 2 System (22) with the LQRI control $u=\tilde{L} x$ is asymptotically stable and $J_{\infty} \leq \tilde{V}\left(\eta_{0}\right)$. Given matrices $\tilde{Q}>0, \tilde{R}>0$. If there exist matrices $\tilde{S}, \tilde{Y}$ such that

$\min _{\gamma_{+}, \bar{Y}, \bar{S}} \gamma_{+}$subject to

$$
\begin{gathered}
{\left[\begin{array}{ccc}
(\tilde{A} \bar{Y}+\tilde{B} \bar{S})+(\tilde{A} \bar{Y}+\tilde{B} \bar{S})^{t} & \tilde{Y} \tilde{Q} & \overline{Y L}^{t} \tilde{R} \\
\bullet & -\tilde{Q} & 0 \\
\bullet & \bullet & -\tilde{R}
\end{array}\right]<0} \\
{\left[\begin{array}{cc}
\gamma_{+} & x_{o}^{t} \\
\bullet & \bar{Y}
\end{array}\right] \geq 0}
\end{gathered}
$$

has a feasible solution, then LQRI gain matrix is $\tilde{L}=\bar{S} \bar{Y}^{-1}$.

Proof. By Assumption (2), and using control $u=\tilde{L} x$ in system (22), the inequality of the derivative of the Lyapunov functional is expressed as

$$
x^{t}\left[\bar{K}(\tilde{A}+\tilde{B} \tilde{L})+(\tilde{A}+\tilde{B} \tilde{L})^{t} \bar{K}^{t}\right] x \leq-x^{t}[\tilde{Q}+\tilde{L} t \tilde{R} \tilde{L}] x
$$

It is evident that (26) is satisfied if there exists $\tilde{L}$ and $\bar{K}$ such that

$$
\bar{K}(\tilde{A}+\tilde{A} \tilde{L})+(\tilde{A}+\tilde{A} \tilde{L})^{t} \bar{K}^{t}-\left[\tilde{Q}+\tilde{L}^{t} \tilde{R} \tilde{L}\right] \leq 0
$$

Simple computations on (13) in view of Assumption (1) yields $J_{\infty} \leq \tilde{V}\left(x_{0}\right)$. By minimizing the upper bound $\gamma_{+}$on the cost $x_{0}^{t} K x_{0}$, we obtain

$$
\min _{\gamma_{+\bar{K}, \tilde{L}}} \gamma_{+} \text {subject to }(27)
$$


To convexify the above problem, we first express (27) as

$$
\begin{gathered}
\Phi=\bar{K}(\tilde{A}+\tilde{B} \tilde{L})+(\tilde{A}+\tilde{B} \tilde{L})^{t} \bar{K}^{t} \\
\Pi=\left[\begin{array}{ccc}
\Phi & \tilde{Q} & \tilde{L}^{t} R \\
\bullet & -\tilde{Q} & 0 \\
\bullet & \bullet & -\tilde{R}
\end{array}\right] \leq 0
\end{gathered}
$$

Pre- and post-multiply (29) by $\operatorname{diag}\{\bar{Y}, I, I\}$ and using $\bar{Y}=\bar{K}^{-1}, \bar{S}=L \bar{K}^{-1}$ it yields (24). Additionally, the inequality bound of the Lyapunov functional can be expressed as

$$
\left[\begin{array}{cc}
\gamma_{+} & x_{o}^{t} \\
\bullet & \bar{K}^{-1}
\end{array}\right] \geq 0
$$

which can be manipulated to yield (25). When a feasible solution is attained, we get $\tilde{L}=\bar{S} \bar{Y}^{-1}, \bar{K}=\bar{Y}^{-1}$ as desired.

Remark 1. labelremA The results of Theorems 1-2 establish improved tools for control designer since on one hand it affords the computational effectiveness in determining the feedback gain matrix and the associated cost. On the other hand, it gives an opportunity to compromise between design efficiency and performance.

\subsection{Model Predictive Control}

The model predictive controller performs a discrete-time optimization of of the form [12]:

$$
\begin{gathered}
x(k+1)=A x(k)+B u(k) \\
y(k)=C x(k) \\
\min J=x^{t}\left(k+n_{p}\right) S x\left(k+n_{p}\right)+\sum_{i=0}^{n_{p}-1} x(k+i)^{t} Q x(k+i)+u(k+i)^{t} R u(k+i)
\end{gathered}
$$

where $x_{k} \in \mathfrak{R}^{n}, \quad u_{k} \in \mathfrak{R}^{m}, \quad y_{k} \in \mathfrak{R}^{p}, A \in \mathfrak{R}^{n \times n}, \quad B \in \mathfrak{R}^{n \times m}$ and $C \in \mathfrak{R}^{q \times n}$. It is desired to synthesize a controller of the form:

$$
\begin{gathered}
x_{c}(k+1)=A_{c} x(k)+B_{c} u(k) \\
y_{c}(k)=C_{c} x_{c}(k)
\end{gathered}
$$

that stabilizes the closed loop system. The closed loop system can be represented as:

$$
\begin{gathered}
\zeta(k+1)=\bar{A} \zeta(k)+\bar{B} u(k) \\
u(k)=K \zeta(k), y(k)=\bar{C} \zeta(k), \bar{B}=\left[\begin{array}{c}
B \\
0
\end{array}\right] \\
\zeta=\left[\begin{array}{c}
x(k) \\
x_{c}(k)
\end{array}\right], \bar{A}=\left[\begin{array}{cc}
A & 0 \\
B_{c} & A_{c}
\end{array}\right] \\
\bar{C}=\left[\begin{array}{ll}
C & 0
\end{array}\right], \bar{K}=\left[\begin{array}{ll}
0 & C_{c}
\end{array}\right]
\end{gathered}
$$

We now consider infinite-time horizon formulation of (32) with 


$$
\begin{aligned}
R>0,0 \leq \bar{Q}=\operatorname{diag}\left[\begin{array}{ll}
Q & 0
\end{array}\right]: & \\
J & =\sum_{i=0}^{\infty} \zeta(k+i)^{t} \bar{Q} \zeta(k+i)+u(k+i)^{t} \bar{R} u(k+i)
\end{aligned}
$$

Again, we define the quadratic Lyapunov function, $V(x)=\zeta^{T} P_{+} \zeta, P_{+}>0$ such that

$$
V(x(k+i+1))-V(x(k+i))<-\zeta(k+i)^{\mathrm{T}} \bar{Q} \zeta(k+i)-u(k+i)^{\mathrm{T}} R u(k+i)
$$

The problem is thus cast into the LMI form:

$$
\begin{aligned}
& \max \gamma_{+}^{-1} \text { s.t. } \\
& {\left[\begin{array}{ll}
Y & I \\
I & X
\end{array}\right]-\gamma^{-1} \phi>0} \\
& {\left[\begin{array}{cccccc}
Y & I & \Gamma & Z & 0 & 0 \\
\bullet & X & A & \Delta & 0 & 0 \\
\bullet & \bullet & \Pi & \Omega & 0 & 0 \\
\bullet & \bullet & \bullet & X & L^{\mathrm{T}} & X Q^{1 / 2} \\
\bullet & \bullet & \bullet & \bullet & R^{-1} & 0 \\
\bullet & \bullet & \bullet & \bullet & \bullet & 0
\end{array}\right]>0} \\
& {\left[\begin{array}{ccc}
\gamma^{-1} u_{\max }^{2} I & 0 & L \\
\bullet & Y & I \\
\bullet & \bullet & X
\end{array}\right]>0,\left[\begin{array}{ccc}
Y & I & A^{\mathrm{T}} C^{\mathrm{T}} \\
? & X & (A X+C B L)^{\mathrm{T}} \\
\bullet & \bullet & \gamma^{-1} y_{\max }^{2} I
\end{array}\right]>}
\end{aligned}
$$

$\gamma=Y A+F C, \quad \Delta=A X+B L, \quad \Pi=Y-Q, \Omega=I-Q X \quad$ When a feasible solution to (37) is obtained as $V=(I-Y X)\left(U^{\mathrm{T}}\right)^{-1}, \quad C_{c}=L\left(U^{\mathrm{T}}\right)^{-1}, \quad B_{c}=V^{-1} F$ and $A_{c}=V^{-1} Z\left(U^{\mathrm{T}}\right)^{-1}$.

Remark 2. labelremD The controllers designed in the foregoing sections enjoy the following features.

- The feedback gains are computed from the feasible solution of convex optimization problems over linear matrix inequalities for which the LMI-solver of Matlab provides an effective software support.

- The realization of the feedback gains is simple and readily programmed in microprocessor ships.

- The developed designs are easily reproducible, repeated and fine-tuned whenever needed.

\section{Simulation Studies}

In this section, we report on the results obtained from wind turbine emulation available at the Distributed Control Research Lab (DCRL). We consider data from the National Renewable Energy Laboratory (NREL) $5 \mathrm{MW}$ offshore wind turbine [10] and incorporated in an emulator experimental setup, see Figure 2. The model consist of a two-mass dive drain, three blades, a pitch and generator torque actuator with parameters listed in Table 1 . The experiment development 
focuses on horizontal-axis wind turbine emulation by using commonly available electric drives laboratory components, including Matlab7, dSPACE8 ControlDesk, Permanent Magnet DC machines, as well as associated pre-lab analysis based on the controller designs. Three controllers: LQR, LQRI (LQR with Integral action) and the MPC (Model Predictive Controller) were applied to the system.

The ensuing results are depicted in Figures 3-7, from which it is evident that the LQRI outperforms the other controllers when the system is subjected to a step change in wind velocity.

\section{Conclusion}

Three control algorithms: LQR, LQRI and MPC have been compared for two

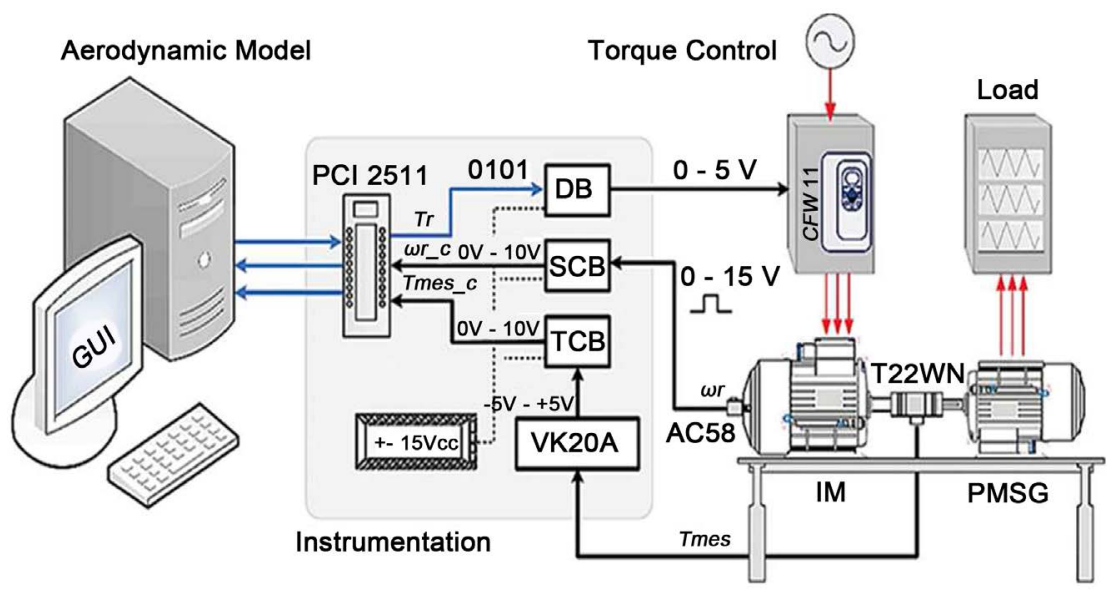

Figure 2. Representation of the wind turbine emulator experimental setup.
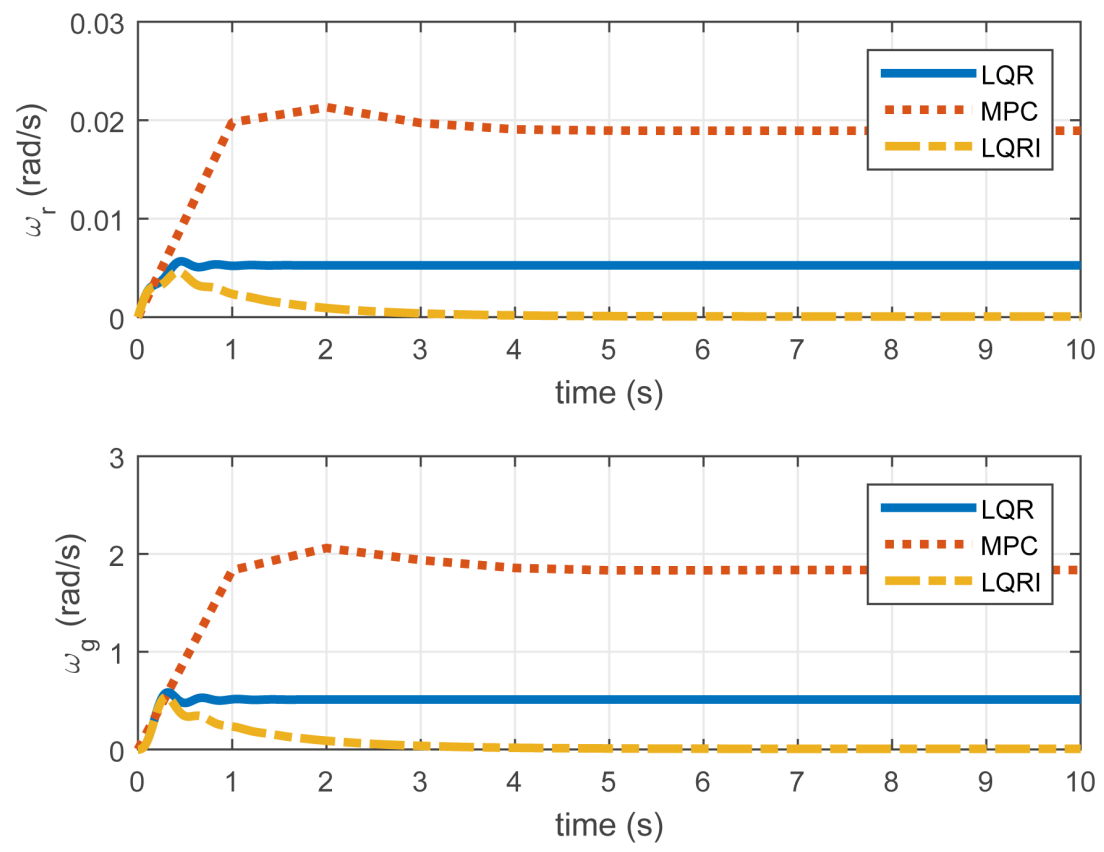

Figure 3. Response of rotor angular speed $\omega_{r}$ and generator angular speed $\omega_{g}$. 

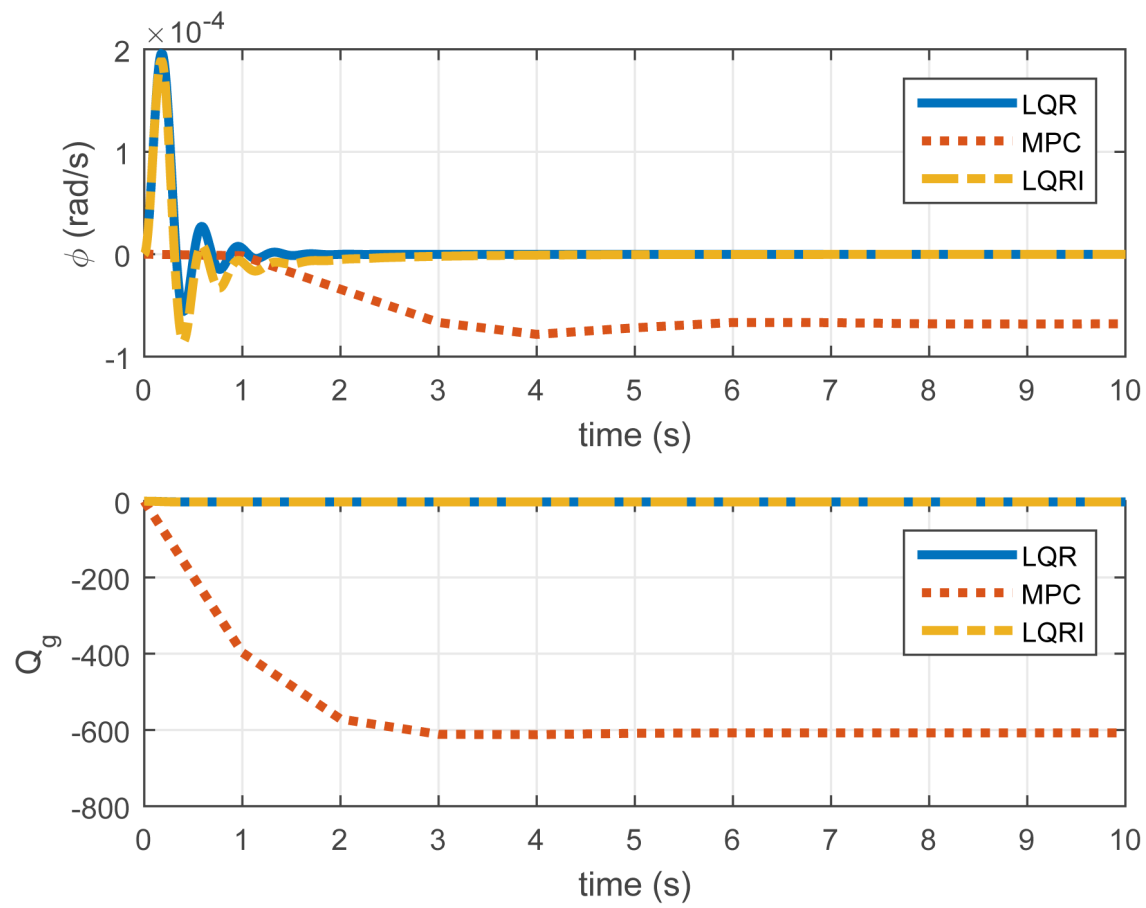

Figure 4. Response of torsional speed $\phi$ and generator torque.
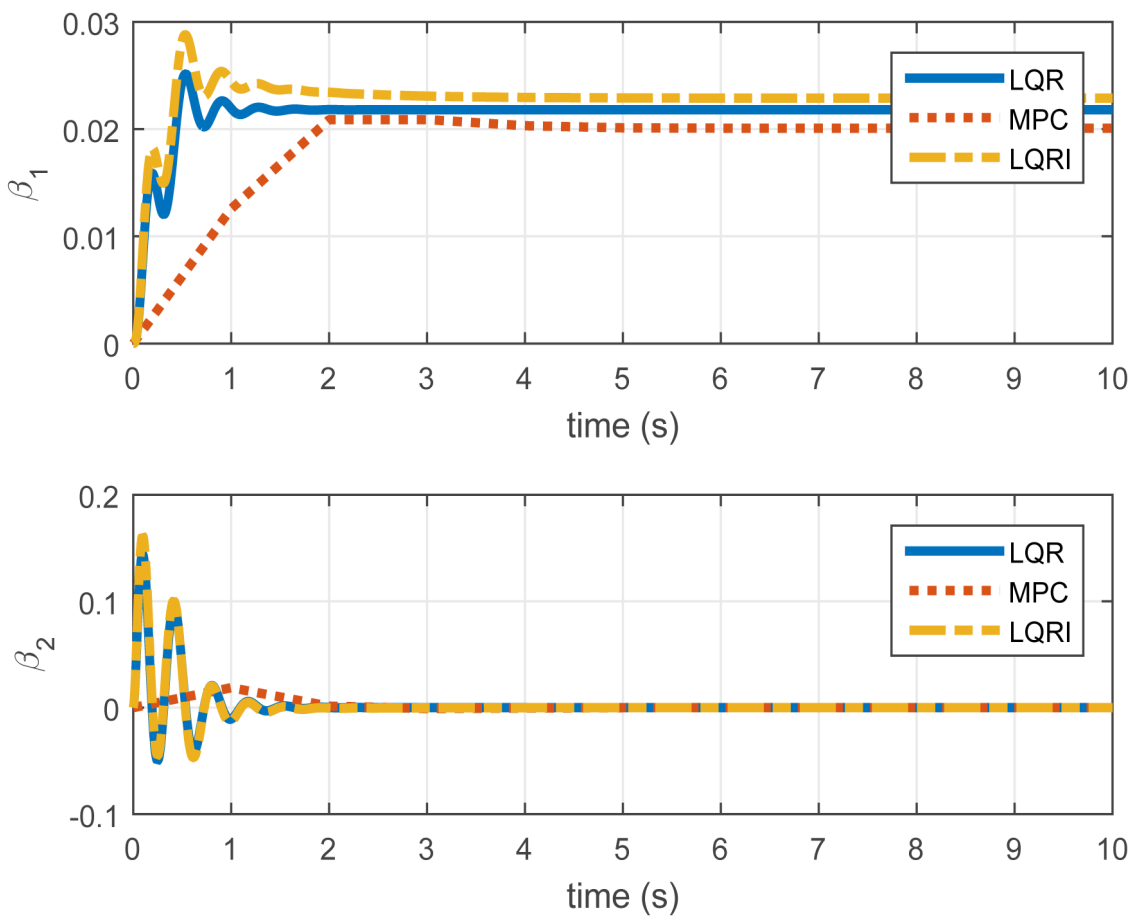

Figure 5. Pitch displacement $\beta_{1}$ and pitch velocity $\beta_{2}$.

different wind turbine models. For system 1, the LQRI was better at rejecting wind velocity disturbances. The same responses were observed in system 2 for both LQR and LQRI controllers with both performing better than the MPC controllers. 


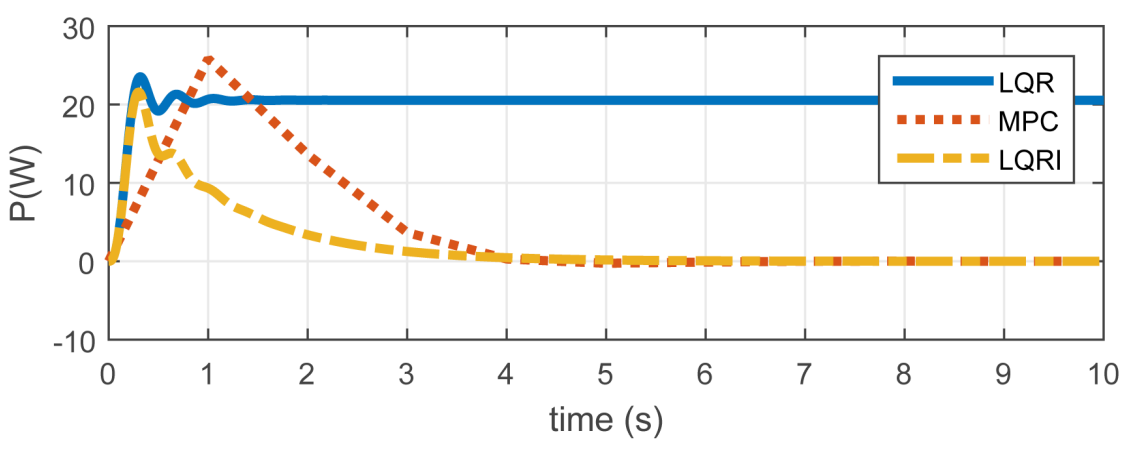

Figure 6. Generator output power.
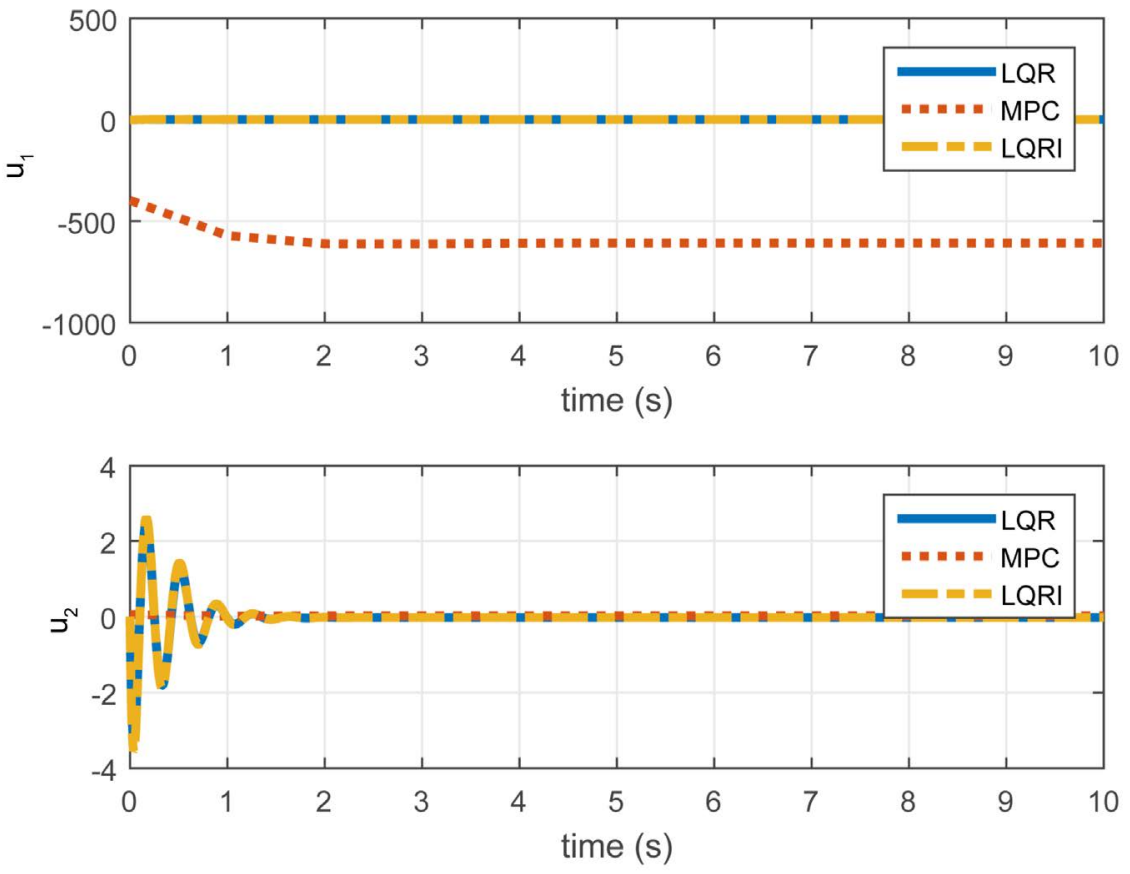

Figure 7. Control effort.

\section{Acknowledgments}

We thank the Editor and the referee for their comments. This work is supported by the deanship for scientific research (DSR) at KFUPM through distinguished professorship award project no. IN 141003.

\section{References}

[1] Tummala, A., Velamati, R.K., Sinha, D.K., Indraja, V. and Krishna, V.H. (2016) A Review on Small Scale Wind Turbines. Renewable and Sustainable Energy Reviews, 56, 1351-1371. https://doi.org/10.1016/j.rser.2015.12.027

[2] Mercado-Vargas, M., Gómez-Lorente, D., Rabaza, O. and Alameda-Hernandez, E. (2015) Aggregated Models of Permanent Magnet Synchronous Generators Wind Farms. Renewable Energy, 83, 1287- 1298.

https://doi.org/10.1016/j.renene.2015.04.040

[3] Hassine, I.M.-B., Naouar, M.W. and Mrabet-Bellaaj, N. (2015) Predictive Control Strategies for Wind Turbine System Based on Permanent Magnet Synchronous Ge- 
nerator. ISA Transactions, 62, 73-80. https://doi.org/10.1016/j.isatra.2015.12.002

[4] Yan, J., Lin, H., Feng, Y. and Zhu, Z. (2014) Control of a Grid-Connected DirectDrive Wind Energy Conversion System. Renewable Energy, 66, 371-380. https://doi.org/10.1016/j.renene.2013.12.037

[5] Tahir, K., Belfedal, C., Allaoui, T. and Champenois, G. (2016) A New Control Strategy of WFSG-Based Wind Turbine to Enhance the $\{$ LVRT $\}$ Capability. International Journal of Electrical Power \& Energy Systems, 79, 172-187. https://doi.org/10.1016/j.ijepes.2016.01.008

[6] Datta, S., Mishra, J.P. and Roy, A.K. (2015) Modified Speed Sensor-Less Grid Connected DFIG Based Wind Energy Conversion System for Decoupled Control of Active and Reactive Power. 2015 International Conference on Power and Advanced Control Engineering (ICPACE), Bengalooru, 12-14 August 2015, 28-35.

https://doi.org/10.1109/ICPACE.2015.7274912

[7] Zamanifar, M., Fani, B., Golshan, M. and Karshenas, H. (2014) Dynamic Modeling and Optimal Control of $\{$ DFIG $\}$ Wind Energy Systems Using $\{$ DFT $\}$ and NSGA-II. Electric Power Systems Research, 108, 50-58.

https://doi.org/10.1016/j.epsr.2013.10.021

[8] Bossoufi, B., Karim, M., Lagrioui, A., Taoussi, M. and Derouich, A. (2015) Observer Backstepping Control of DFIG-Generators for Wind Turbines Variable-Speed: FPGA-Based Implementation. Renewable Energy, 81, 903-917. https://doi.org/10.1016/j.renene.2015.04.013

[9] Nasiri, M., Milimonfared, J. and Fathi, S. (2015) A Review of Low-Voltage RideThrough Enhancement Methods for Permanent Magnet Synchronous Generator Based Wind Turbines. Renewable and Sustainable Energy Reviews, 47, 399-415. https://doi.org/10.1016/j.rser.2015.03.079

[10] Gosk, A. (2011) Model Predictive Control of a Wind Turbine. Master's Thesis, Technical University of Denmark, Denmark.

[11] Anderson, B.D.O. and Moore, J.B. (1971) Linear Optimal Control. Prentice-Hall, Inc., Englewood Cliffs.

[12] Granado, E., Colmenares, W., Bernumssan, J. and Garcia, G. (2002) LMI Based MPC. 15th Triennial World Congress, Barcelona, 21-26 July 2002. https://doi.org/10.3182/20020721-6-es-1901.00598

\section{Submit or recommend next manuscript to SCIRP and we will provide best service for you:}

Accepting pre-submission inquiries through Email, Facebook, LinkedIn, Twitter, etc. A wide selection of journals (inclusive of 9 subjects, more than 200 journals) Providing 24-hour high-quality service User-friendly online submission system Fair and swift peer-review system Efficient typesetting and proofreading procedure Display of the result of downloads and visits, as well as the number of cited articles Maximum dissemination of your research work

Submit your manuscript at: http://papersubmission.scirp.org/

Or contactica@scirp.org 